NBER WORKING PAPER SERIES

\title{
A FRAMEWORK FOR EXPLORING THE MACROECONOMIC DETERMINANTS OF SYSTEMATIC RISK
}

\author{
Torben G. Andersen \\ Tim Bollerslev \\ Francis X. Diebold \\ Jin (Ginger) Wu \\ Working Paper 11134 \\ http://www.nber.org/papers/w11134
}

\author{
NATIONAL BUREAU OF ECONOMIC RESEARCH \\ 1050 Massachusetts Avenue \\ Cambridge, MA 02138 \\ February 2005
}

The views expressed herein are those of the author(s) and do not necessarily reflect the views of the National Bureau of Economic Research.

(C) 2005 by Torben G. Andersen, Tim Bollerslev, Francis X. Diebold, and Jin (Ginger) Wu. All rights reserved. Short sections of text, not to exceed two paragraphs, may be quoted without explicit permission provided that full credit, including $\odot$ notice, is given to the source. 
A Framework for Exploring the Macroeconomic Determinants of Systematic Risk Torben G. Andersen, Tim Bollerslev, Francis X. Diebold, and Jin (Ginger) Wu NBER Working Paper No. 11134

February 2005

JEL No. G12

\begin{abstract}
We selectively survey, unify and extend the literature on realized volatility of financial asset returns.

Rather than focusing exclusively on characterizing the properties of realized volatility, we progress by examining economically interesting functions of realized volatility, namely realized betas for equity portfolios, relating them both to their underlying realized variance and covariance parts and to underlying macroeconomic fundamentals.
\end{abstract}

Torben G. Andersen

Kellogg School of Management

Northwestern University

2001 Sheridan Road

Evanston, IL 60208

and NBER

t-andersen@kellogg.northwestern.edu

Tim Bollerslev

Department of Economics

Duke University

Box 90097

Durham, NC 27708-0097

and NBER

boller@econ.duke.edu
Francis X. Diebold

Department of Economics

University of Pennsylvania

3718 Locust Walk

Philadelphia, PA 19104-6297

and NBER

fdiebold@sas.upenn.edu

Jin (Ginger) Wu

Department of Economics

University of Pennsylvania

3718 Locust Walk

Philadelphia, PA 19104-6297

jinw@ssc.upenn.edu 


\title{
A Framework for Exploring the Macroeconomic Determinants of Systematic Risk
}

\author{
By Torben G. Andersen, Tim Bollerslev, Francis X. Diebold, And Jin (Ginger) Wu*
}

The increasing availability of high-frequency asset return data has had a fundamental impact on empirical financial economics, focusing attention on asset return volatility and correlation dynamics, with key applications in portfolio and risk management. So-called "realized" volatilities and correlations have featured prominently in the recent literature, and numerous studies have provided direct characterizations of the unconditional and conditional distributions of realized volatilities and correlations across different assets, asset classes, countries, and sample periods. For overviews see Torben G. Andersen, Tim Bollerslev, Peter F. Christoffersen and Francis X. Diebold (2005a, b).

In this paper we selectively survey, unify and extend that literature. Rather than focusing exclusively on characterization of the properties of realized volatility, we progress by examining economically interesting functions of realized volatility, namely realized betas for equity portfolios, relating them both to their underlying realized variance and covariance parts and to underlying macroeconomic fundamentals.

We proceed as follows. In part I we introduce realized volatility and basic theoretical results concerning its convergence to integrated volatility. In part II we move to realized beta and characterize its dynamics relative to those of its variance and covariance components. In part III we introduce a state space representation that facilitates extraction and prediction of true (latent) betas based on their realized values, and which also allows for simple incorporation and joint modeling of macroeconomic fundamentals. In part IV we provide an illustrative empirical example, and we conclude in part V.

\section{Realized Volatility}


Let the $N \times 1$ logarithmic vector price process, $p_{t}$, follow a multivariate continuous-time stochastic

volatility diffusion,

$$
d p_{t}=\mu_{t} d t+\Omega_{t} d W_{t}
$$

where $W_{t}$ denotes a standard $N$-dimensional Brownian motion, both the $N \times N$ positive definite diffusion matrix, $\Omega_{t}$ , and the $N$-dimensional instantaneous drift, $\mu_{t}$, are strictly stationary and jointly independent of $W_{t}$ (extensions to allow for leverage effects, or non-zero correlations between $W_{t}$ and $\Omega_{t}$, and/or jumps in the price process could in principle be incorporated as well). Also, suppose that the $N^{\prime}$ th element of $p_{t}$ contains the log price of the market, and the $i$ 'th element of $p_{t}$ contains the $\log$ price of the $i$ 'th individual stock, so that the corresponding covariance matrix contains both the market variance, say $\sigma_{M, t}^{2}=\Omega_{(N N), t}$, and the individual equity covariance with the market, say $\sigma_{i M, t}=\Omega_{(i N), t}$.

Conditional on the realized sample paths of $\mu_{t}$ and $\Omega_{t}$, the distribution of the continuously compounded $h$ period return, $r_{t+h, h} \equiv p_{t+h}-p_{t}$, is then

$$
r_{t+h, h} / \sigma\left\{\mu_{t+\tau}, \Omega_{t+\tau}\right\}_{\tau=0}^{h} \sim N\left(\int_{0}^{h} \mu_{t+\tau} d \tau, \int_{0}^{h} \Omega_{t+\tau} d \tau\right)
$$

where $\sigma\left\{\mu_{t+\tau}, \Omega_{t+\tau}\right\}_{\tau=0}^{h}$ denotes the $\sigma$-field generated by the sample paths of $\mu_{t+\tau}$ and $\Omega_{t+\tau}$ for $0 \leq \tau \leq h$. The integrated diffusion matrix $\int_{0}^{h} \Omega_{t+\tau} d \tau$ therefore provides a natural measure of the true latent $h$-period volatility. Under weak regularity conditions, it follows from the theory of quadratic variation that

$$
\Sigma_{j=1, \ldots,[h / \Delta]} r_{t+j \cdot \Delta, \Delta} \cdot r_{t+j \cdot \Delta, \Delta}^{\prime}-\int_{0}^{h} \Omega_{t+\tau} d \tau \rightarrow 0
$$

almost surely (a.s.) for all $t$ as the return sampling frequency increases $(\Delta \rightarrow 0)$. Thus, by using sufficiently finelysampled high-frequency returns, it is possible in theory to construct a realized diffusion matrix that is arbitrarily 
close to the integrated diffusion matrix (for a survey of the relevant theory, see Andersen, Bollerslev and Diebold, 2005). In practice, market microstructure frictions limits the highest feasible sampling frequency $(\Delta \geq \delta>0)$, and the best way to deal with this, whether using the simple estimator in (3) or some variant thereof, is currently a very active area of research.

Meanwhile, key empirical findings for realized volatility include lognormality and long memory of volatilities and correlations (Andersen, Bollerslev, Diebold and Paul Labys, 2001; Andersen, Bollerslev, Diebold and Heiko Ebens, 2001), as well as normality of returns standardized by realized volatility (Andersen, Bollerslev, Diebold and Labys, 2000). Those properties, as distilled in the lognormal / normal mixture model of Andersen, Bollerslev, Diebold and Labys (2003), have important implications for risk management and asset allocation.

\section{Realized Beta and its Components}

Although characterizations of the properties of realized variances and covariances are of interest, alternative objects are often of greater economic significance with a leading example being the market beta of a portfolio. If either the market volatility or its covariance with portfolio returns is time-varying, then the portfolio beta will generally be time-varying. Hence it is clearly of interest to explore the links between time-varying volatilities, time-varying correlations, and time-varying betas. One may construct realized betas from underlying realized covariance and variance components, or conversely, decompose realized betas into realized variance and covariance components.

Armed with the relevant realized market variance and realized covariance measures, we can readily define and empirically construct "realized betas." Using an initial subscript to indicate the corresponding element of a 
vector, we denote the realized market volatility by

$$
\hat{v}_{M, t, t+h}^{2}=\Sigma_{j=1, \ldots,[h / \Delta]} r_{(N), t+j \cdot \Delta, \Delta}^{2}
$$

and the realized covariance between the market and the $i$ th portfolio return by

$$
\hat{v}_{i M, t, t+h}=\sum_{j=1, \ldots,[h / \Delta]} r_{(i), t+j \cdot \Delta, \Delta} \cdot r_{(N), t+j \cdot \Delta, \Delta} \cdot
$$

Now defining the realized beta as the ratio between the two, it follows under the assumptions above that

$$
\hat{\beta}_{i, t, t+h} \equiv \frac{\hat{v}_{i M, t, t+h}}{\hat{v}_{M, t, t+h}^{2}} \rightarrow \beta_{i, t, t+h} \equiv \int_{0}^{h} \Omega_{(i N), t+\tau} d \tau / \int_{0}^{h} \Omega_{(N N), t+\tau} d \tau,
$$

a.s. for all $t$ as $\Delta \rightarrow 0$, so that realized beta is consistent for the corresponding true integrated beta.

By comparing the properties of directly-measured betas to those of directly-measured variances and covariances, we can decompose movements in betas in informative ways. In particular, because the long memory in underlying variances and covariances may be common, it is possible that betas may be only weakly persistent (short-memory, $I(d)$, with $d \approx 0$ ), despite the widespread finding that realized variances and covariances are longmemory (fractionally- integrated, $I(d)$, with $d \approx 0.4$ ). Recent work by Andersen, Bollerslev, Diebold and Ginger $\mathrm{Wu}(2005 \mathrm{a})$ indicates that the relevant realized variances and covariances are indeed reasonably wellcharacterized as nonlinearly fractionally cointegrated in this fashion (as beta is an a priori known ratio of the two measures).

\section{A State Space Framework Facilitating the Inclusion of Macroeconomic Fundamentals}

Although the decomposition of realized betas into contributions from underlying variances and covariances is intriguing, a more thorough economic analysis would seek to identify the fundamental determinants of realized variances and covariances that impact realized betas. Here we take some steps in that 
direction, directly allowing for dependence of betas on underlying macroeconomic fundamentals.

First, in parallel to the volatility model in Ole Barndorff-Nielsen and Neil Shephard (2002), the timevarying integrated/realized beta may be conveniently cast in state space form. The realized beta equals the true latent integrated beta, plus a weak white noise measurement error, asymptotically Gaussian in the sampling frequency $(\Delta \rightarrow 0)$. Normalizing $h \equiv 1$ and suppressing the subscripts:

$$
\hat{\beta}_{t}=\beta_{t}+u_{t}
$$

We can easily allow for dynamics in $\beta_{t}$, as exemplified by the first-order autoregressive representation

$$
\beta_{t}=\gamma_{0}+\gamma_{1} \beta_{t-1}+v_{t}
$$

where $v_{t}$ is weak white noise. We therefore have a state space system, with measurement equation (7a) and transition equation ( $7 \mathrm{~b}$ ), so that the Kalman filter may be used for extraction and prediction of the latent integrated $\boldsymbol{\beta}_{t}$ based on the observed $\hat{\boldsymbol{\beta}}_{t}$ (a more refined approach in which the nonconstant variance of $\boldsymbol{u}_{t}$ is equated to the asymptotic, for $\Delta \rightarrow 0$, expression in Barndorff-Nielsen and Shephard, 2004, could also be applied). Note, that the system in $(7 a, b)$ is distinctly different from the one in which the measurement equation is replaced by a conditional CAPM model, $r_{t}=\alpha+\beta_{t} r_{M, t}+\varepsilon_{t}$ (see, e.g., Andrew Ang and Josephn Chen, 2004, and Gergana Jostova and Alexander Philipov, 2005, and the references therein. For an alternative intraday based beta estimation procedure, see, e.g., Qianqiu Liu, 2003). The smoothed version of $\beta_{t}$ extracted by the Kalman filter from $(7 a, b)$, in particular, should compare favorably to the standard practice of assuming that the sampling frequency is so high that $\hat{\boldsymbol{\beta}}_{t}$ is effectively indistinguishable from $\boldsymbol{\beta}_{t}$, or $\boldsymbol{u}_{\boldsymbol{t}} \approx 0$ (See also Dean Foster and Dan Nelson, 1996, who argue for smoothing of realized betas, from a very different and complementary perspective). 
Second, note that we may readily include macroeconomic fundamentals in the state space dynamics, by augmenting the state vector as in the system:

$$
\begin{aligned}
& \hat{\beta}_{t}=z^{\prime} B_{t}+u_{t} \\
& B_{t}=\Gamma_{0}+\Gamma_{1} B_{t-1}+v_{t},
\end{aligned}
$$

where $z^{\prime} \equiv(1,0, \ldots, 0), u_{t} \sim\left(0, \sigma_{u}^{2}\right), \Gamma_{0}$ is a vector of intercepts, $\Gamma_{1}$ is a matrix of coefficients, $B_{t}^{\prime} \equiv\left(\beta_{t}, x_{t}^{\prime}\right), x_{t}$ is a column vector of macroeconomic variables, and $v_{t} \sim(0, \Sigma)$ is a vector of transition disturbances. The vector autoregressive transition equation (8b) permits interaction between beta and macroeconomic fundamentals, both dynamically (via $\Gamma_{1}$ ) and contemporaneously (via the covariances in $\mathbf{\Sigma}$ ). For illustration, in this paper, we only explore macroeconomic indicators one at a time, under an assumption of recursive transition dynamics. That is, letting $\Sigma=\operatorname{diag}\left(\sigma_{v 1}^{2}, \sigma_{v 2}^{2}\right)$, we estimate the system

$$
\begin{aligned}
& \hat{\beta}_{t}=\beta_{t}+u_{t} \\
& \left(\begin{array}{l}
\beta_{t} \\
x_{t}
\end{array}\right)=\left(\begin{array}{l}
\gamma_{01} \\
\gamma_{02}
\end{array}\right)+\left(\begin{array}{cc}
\gamma_{11} & \gamma_{12} \\
0 & \gamma_{22}
\end{array}\right)\left(\begin{array}{l}
\beta_{t-1} \\
x_{t-1}
\end{array}\right)+\left(\begin{array}{l}
v_{1 t} \\
v_{2 t}
\end{array}\right) .
\end{aligned}
$$

For simplicity, we further assume homoskedastic measurement errors for monthly realized betas. This is clearly not true for daily data, but a more palatable approximation at the monthly level that is relevant for the analysis below. It follows that inference based on the standard Kalman Filter is valid.

\section{An Illustrative Application}

We use underlying fifteen-minute returns for individual NYSE-listed stocks and the value-weighted market portfolio. We construct all returns from the TAQ dataset, February 1, 1993 through May 31, 2003, excluding real estate investment trusts, stocks of companies incorporated outside the United States, and closed- 
end mutual funds. Next, we sort the firms into twenty-five portfolios, corresponding to various combinations of the five market capitalization ("size") and five book-to-market ("value") quintiles, month-by-month, re-balancing each month. We denote the twenty-five portfolios by $i j$, for $i, j=1, \ldots, 5$, where $i$ refers to size quintile and $j$ refers to value quintile (from low to high). Finally, for each of the twenty-five portfolios, we use the fifteenminute portfolio and market returns to construct monthly realized covariances of each portfolio return with the market return, the realized variance of the market return, and the ratio, or "realized beta." To adjust for asynchronous trading, we use an equally-weighted average of contemporaneous realized beta and four leads and lags.

In Figure 1, we show extractions of the latent integrated betas obtained using the Kalman smoother. Substantial and highly-persistent time variation is evident for all the realized betas, but they do not appear to be trending or otherwise nonstationary; instead reverting to fixed means. We have also shaded the March-November 2001 recession for visual reference. Looking across the columns from low- to high-value portfolios, the betas for many portfolios appear to increase substantially during and around the recession, and the high-value portfolio betas seem to be more responsive over the cycle.

We now assess these graphically-motivated conjectures more rigorously by estimating the time-varying beta model in (9a,b), explicitly allowing for macroeconomic influences. In Andersen, Bollerslev, Diebold and Wu (2005b), we study all twenty-five portfolios and several macroeconomic indicators, alone and in combination, including industrial production, the term premium, the default premium, the consumption/wealth ratio, the consumer price index, and the consumer confidence index. Here we merely sketch some illustrative results, 
focusing on representative large-capitalization portfolios 51, 53 and 55, and a central macroeconomic indicator, industrial production growth $(I P)$.

We display the estimation results in Table 1. The $\beta$ own-lag coefficients $\gamma_{11}$ indicate substantial own persistence, while the $I P$ own-lag coefficients $\gamma_{22}$ are obviously much smaller. This is natural as the $I P$ variable is a growth rate (change in logarithm). The key macro-finance interaction coefficient, $\gamma_{12}$, summarizes the response of $\boldsymbol{\beta}_{t}$ to movements in $I P_{t-1}$. Interestingly, and in keeping with our earlier conjecture, both the statistical and economic significance of the estimates of $\gamma_{12}$ increase with value, as measured by book-to-market. For portfolio 51, the point estimate of $\gamma_{12}$ is near zero and statistically insignificant at any conventional level, while for portfolio 53, the point estimate is substantially larger in magnitude (-3.4) and significant at the ten percent level. For portfolio 55, the point estimate is statistically significant at the one percent level, and quite large at -6.1 , implying that an additional percentage point of $I P_{t-1}$ growth produces a -.061 decrease in $\boldsymbol{\beta}_{55, t}$. Hence as $I P_{t-1}$ varies over the cycle from, say, -0.05 to $+0.05, \beta_{55, t}$ will move substantially. Impulse response functions provide a more complete distillation of the dynamic response patterns.

Although the recursive structure automatically identifies the vector autoregression (10b), we still normalize by the Cholesky factor of $\Sigma$ to express all shocks in standard deviation units. We report results in Figure 2. In parallel with the impact estimates in Table 1, the beta for the growth portfolio 51 shows no dynamic response but, as we move upward through the value spectrum, we find progressively larger effects, with positive $I P_{t-1}$ shocks producing sharp decreases in $\boldsymbol{\beta}_{t}$, followed by very slow reversion to the mean. These are, of course, only partial effects, and a more complete analysis would have to jointly consider the influence of other business cycle 
variables as in $(8 \mathrm{a}, \mathrm{b})$.

\section{Concluding Remarks}

There is an emerging empirical consensus that expected excess returns are counter-cyclical - not only for stocks, as in Martin Lettau and Sydney Ludvigson (2001a), but also for bonds, as in John H. Cochrane and Monika Piazzesi (2005) - whether because risk is higher in recessions, as in George M. Constantinides and Darrell Duffie (1996), or because risk aversion is higher in recessions, as in John Campbell and Cochrane (1999).

The preliminary results reported here indicate that equity market betas do indeed vary with macroeconomic indicators such as industrial production growth, and that the macroeconomic effects on expected returns are large enough to be economically important. Moreover, the preliminary results strongly indicate that the countercyclicality of beta is primarily a value stock phenomenon, suggesting that the well-documented and much-debated value premium (see also the related studies by Andrew Ang and Jun Liu, 2004; Ravi Jagannathan and Zhenyu Wang, 1996; Lettau and Ludvigson, 2001b; Jonathan Lewellen and Stefan Nagel, 2004; Ralitsa Petkova and Lu Zhang, 2004, and the many references therein) may at least in part be explained by an increase in expected returns for value stocks during bad economic times. 


\section{REFERENCES}

Andersen, Torben G.; Bollerslev, Tim; Christoffersen, Peter F. and Diebold, Francis X. "Practical Volatility and Correlation Modeling for Financial Market Risk Management,” in M. Carey and R. Stulz, eds., Risks of Financial Institutions, forthcoming, 2005a.

. "Volatility Forecasting," in G. Elliott, C.W.J. Granger, and A. Timmermann, eds., Handbook of Economic Forecasting. Amsterdam: North-Holland, forthcoming, 2005b.

Andersen, Torben G.; Bollerslev, Tim and Diebold, Francis X. "Parametric and Nonparametric Volatility Measurement," in L.P. Hansen and Y. Ait-Sahalia, eds., Handbook of Financial Econometrics. Amsterdam: North-Holland, forthcoming, 2005.

Andersen, Torben G.; Bollerslev, Tim; Diebold, Francis X. and Ebens, Heiko. “The Distribution of Realized Stock Return Volatility.” Journal of Financial Economics, July 2001, 61(1), pp. 43-76.

Andersen, Torben G.; Bollerslev, Tim; Diebold, Francis X. and Labys, Paul. "Exchange Rate Returns Standardized by Realized Volatility are (Nearly) Gaussian.” Multinational Finance Journal, September/December 2000, 4(3/4), pp. 159-179.

. "The Distribution of Realized Exchange Rate Volatility." Journal of the American Statistical Association, March 2001, 96(453), pp. 42-55. . "Modeling and Forecasting Realized Volatility.” Econometrica, March 2003, 71(2), pp. 579-626.

Andersen, Torben G.; Bollerslev, Tim; Diebold, Francis X. and Wu, Ginger. "Realized Beta: Persistence and Predictability." in T. Fomby, ed., Advances in Econometrics: Econometric Analysis of Economic and 
Financial Time Series, forthcoming, 2005a.

. "Betas and the Macroeconomy." Working Paper in Progress, Northwestern University, Duke University, and University of Pennsylvania, forthcoming, 2005b.

Ang, Andrew amd Liu, Jun. "How to Discount Cashflows with Time-Varying Expected Returns." Journal of Finance, December 2004, 59(6), pp. 2745-2783.

Ang, Andrew and Chen, Joseph. “CAPM over the Long-Run: 1926-2001.” Manuscript, November 2004; Columbia University and University of Southern California.

Barndorff-Nielsen, Ole E. and Shephard, Neil. "Econometric Analysis of Realized Volatility and its Use in Estimating Stochastic Volatility Models.” Journal of the Royal Statistical Society, Series B, Spring 2002, 64(2), pp. 253-280.

. "Econometric Analysis of Realized Covariation: High Frequency Covariance, Regression and Correlation in Financial Economics.” Econometrica, May 2004, 72(3), pp.885-925.

Cochrane, John M. and Piazzesi, Monika. “Bond Risk Premia.” American Economic Review, March 2005, 95(1), forthcoming.

Constantinides, George M. and Duffie, Darrell. “Asset Pricing with Heterogeneous Consumers.” Journal of Political Economy, June 1996, 104(2), pp.219-240.

Foster, Dean P. and Dan B. Nelson. "Continuous Record Asymptotics for Rolling Sample Estimators." Econometrica, January 1996, 64(1), pp.139-174.

Ghysels, Eric and Jacquier, Eric. "Market Beta Dynamics and Portfolio Efficiency.” Manuscript, January $-11-$ 
2005; University of North Carolina at Chapel Hill and HEC, University of Montréal.

Jagannathan, Ravi and Wang, Zhenyu. "The Conditional CAPM and the Cross-Section of Stock Returns."

Journal of Finance, March 1996, 51(1), pp.3-53.

Jostova, Gergana and Philipov, Alexander. "Bayesian Analysis of Stochastic Betas." Journal of Financial and Quantitative Analysis, 2005, forthcoming.

Lettau, Martin and Ludvigson, Sydney. “Consumption, Aggregate Wealth, and Expected Stock Returns.”

Journal of Finance, June 2001a, 56(3), pp.815-849.

Lettau, Martin and Ludvigson, Sydney. "Resurrecting the (C)CAPM: A Cross Sectional test When Risk Premia are Time-Varying.” Journal of Political Economy, 2001b, 109(6), pp.1238-1287.

Lewellen, Jonathan and Nagel, Stefan. "The Conditional CAPM Does Not Explain Asset Pricing Anomalies." Manuscript, January 2004; MIT and Harvard University.

Liu, Qianqiu. “Estimating Betas from High-Frequency Data.” Manuscript, June 2003; Northwestern University. Petkova, Ralitsa and Zhang, Lu. “Is Value Riskier Than Growth?” Manuscript, January 2004; Case Western Reserve University and University of Rochester. 


\section{Footnotes}

*Andersen: Northwestern University, Evanston, IL 60208; Bollerslev: Duke University, Durham, NC 27708;

Diebold and Wu: University of Pennsylvania, Philadelphia, PA 19104. We thank the National Science

Foundation for research support, and Boragan Aruoba, Paul Labys and Heiko Ebens for useful conversations and

productive research collaboration. Finally, we acknowledge discussions with Eric Ghysels, who drew our attention to related ongoing work in Ghysels and Jacquier (2005). 

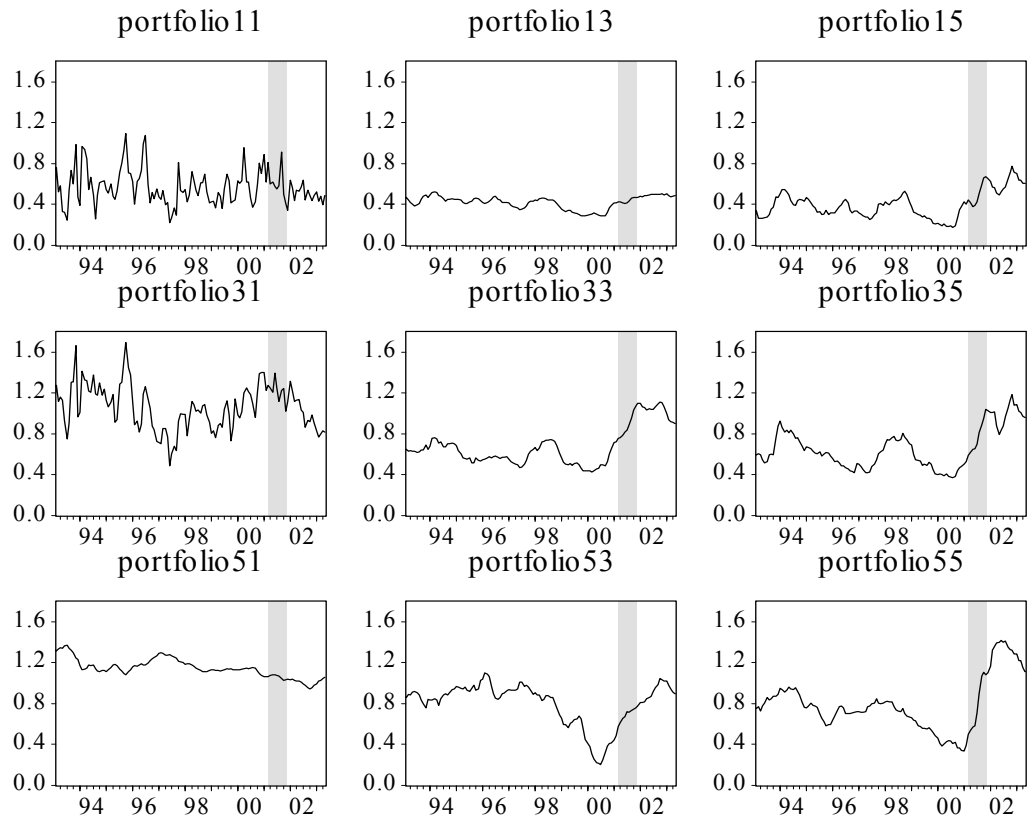

Figure 1. Smoothed Extractions of market Betas, February 1993 to May 2003 

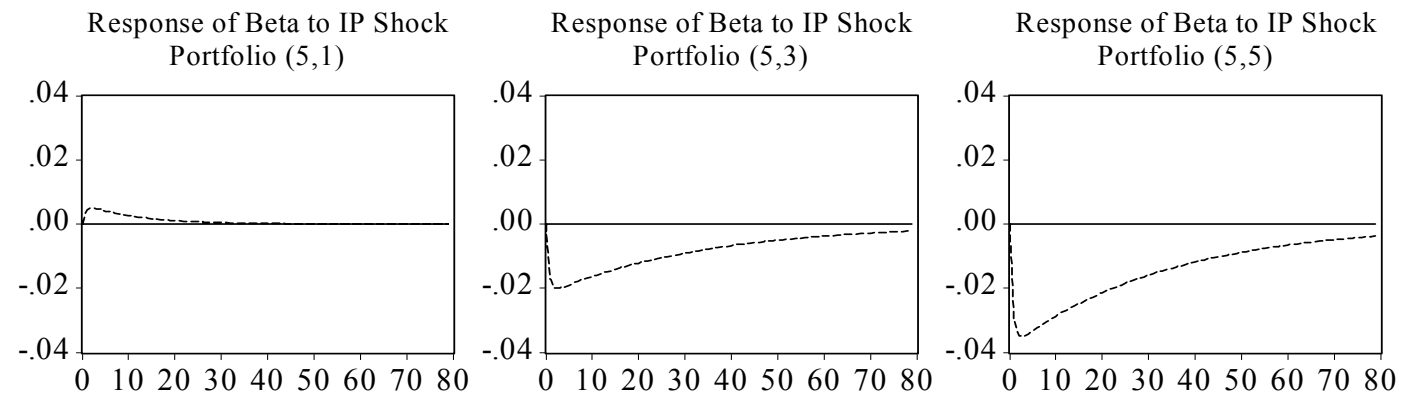

Figure 2. Impulse Response Functions 
Table 1 - Parameter Estimates for Model (10a, b)

\begin{tabular}{llllllll}
\hline \hline & Portfolio 51 & \multicolumn{5}{c}{ Portfolio 53} & \multicolumn{3}{c}{ Portfolio 55 } \\
\cline { 2 - 7 } & Coef. & S.E. & Coef. & S.E. & Coef. & S.E. \\
\hline$\gamma_{01}$ & $0.092^{* *}$ & 0.036 & 0.032 & 0.028 & $0.042^{* *}$ & 0.020 & \\
$\gamma_{02}$ & $0.002^{* * *}$ & 0.0005 & $0.002^{* * *}$ & 0.0005 & $0.002^{* * *}$ & 0.0005 & \\
$\gamma_{11}$ & $0.915^{* * *}$ & 0.031 & $0.971^{* * *}$ & 0.034 & $0.971^{* * *}$ & 0.024 & \\
$\gamma_{12}$ & 0.920 & 1.114 & $-3.486^{*}$ & 2.156 & $-6.101^{* * *}$ & & 1.706 \\
$\gamma_{22}$ & $0.191^{* *}$ & 0.088 & $0.191^{* *}$ & 0.088 & $0.191^{* *}$ & 0.088 & \\
\hline
\end{tabular}

Notes: ${ }^{*}, * *$ and ${ }^{* * *}$ denote statistical significance at the ten percent, five percent and one percent levels, respectively. 